\title{
Um estudo sobre grandezas constitutivas e diferença de pressões arbitrárias em uma suspensão particulada com restrição de incompressibilidade
}

A study on constitutive quantities and difference in arbitrary pressures in a particulate suspension with incompressibility constraint

\author{
D. O. Libório ${ }^{1 *}$; E. J. Santos²; P. L. Santana²; R. L. Pagano²; A. S. Silva ${ }^{3}$ \\ ${ }^{\text {I} P r o g r a m a ~ d e ~ P o ́ s-g r a d u a c ̧ a ̃ o ~ e m ~ E n g e n h a r i a ~ Q u i ́ m i c a, ~ U n i v e r s i d a d e ~ F e d e r a l ~ d e ~ S e r g i p e, ~ 49100-000, ~ S a ̃ o ~ C r i s t o ́ v a ̃ o-~}$ \\ Sergipe, Brasil \\ ${ }^{2}$ Departamento de Engenharia Química, Universidade Federal de Sergipe, 49100-000, São Cristóvão-Sergipe, Brasil \\ ${ }^{3}$ Departamento de Matemática e Programa de Pós-graduação em Engenharia Química, Universidade Federal de \\ Sergipe, 49100-000, São Cristóvão-Sergipe, Brasil
}

*denisson.liborio@gmail.com

(Recebido em 15 de março de 2016; aceito em 06 de abril de 2016)

\begin{abstract}
Este trabalho, no contexto de meio poroso com restrição de incompressibilidade, trata de um estudo sobre grandezas constitutivas e diferença de pressões arbitrárias em uma suspensão particulada. São usadas duas soluções exatas de um sistema de equações do movimento da suspensão particulada, com tais soluções inseridas em modelagem matemática do movimento gravitacional de suspensões particuladas em proveta. A modelagem envolve sub-regiões que variam com o tempo e são delimitadas por interfaces em movimento, inclusive pela onda de aceleração. As soluções exatas e os movimentos da onda e das interfaces, juntamente com dados experimentais da literatura de altura versus tempo da interface superior descendente do próprio teste de proveta, são usados para o cálculo de parâmetros envolvidos em equações propostas para as partes constitutivas da força de interação e de cada tensor tensão. Com isso, é determinada a diferença de pressões arbitrárias. São mostrados resultados numéricos do cálculo dos parâmetros envolvidos nas equações do modelo, usando-se como ponto de partida a determinação dos parâmetros básicos velocidade da onda de aceleração, ponto de encontro da onda com a interface superior descendente e ponto de encontro das interfaces.

Palavras-chave: suspensão particulada, pressões arbitrárias, grandezas constitutivas
\end{abstract}

This work deals with porous media with incompressible constraints and is a study on constitutive quantities and difference in arbitrary pressures in a particulate suspension. In this study, two exact solutions of a system of motion equations of a particulate suspension were used. The solutions were employed in the mathematical modeling of the gravitational motion of particulate suspensions in a test tube. This modeling deals with subregions that vary with time and are bounded by moving interfaces and an acceleration wave. The exact solutions and the motions of waves and interfaces, along with experimental data on height versus time for the descending upper interface of the test tube, as obtained from literature, were used to calculate the parameters involved in the proposed equations for the constituent parts of the force of interaction and of each strain tensor. Thus, the difference in arbitrary pressures was determined. The numerical results of the parameters in the model equations obtained using the basic parameters of acceleration wave velocity, meeting point of the wave with the descending upper interface, and meeting point of interfaces are reported

Keywords: particulate suspension, arbitrary pressures, constitutive quantities

\section{INTRODUÇÃO}

O estudo do movimento de suspensões particuladas é relevante tanto do ponto de vista teórico, como em muitas situações de interesse prático [1-3]

Do ponto de vista de misturas sólido-fluido, a suspensão particulada pode ser estudada com base na teoria contínua de misturas sem reações químicas, que estabelece equações dos balanços de forma mais generalizada, inclusive a desigualdade entrópica que é importante tanto para análise de 
grandezas constitutivas, como também para grandezas arbitrárias quando há algum tipo de restrição [4].

De acordo com Silva e Jesus [4], dependendo de como as grandezas constitutivas e arbitrárias são agrupadas e interpretadas, formas diferentes podem ser obtidas para sistemas de equações do movimento de uma suspensão particulada com restrição de incompressibilidade. A forma do sistema escolhido é aquela que facilita a determinação de soluções exatas, as mais simples possíveis, para serem inseridas em modelagem matemática.

Em uma dimensão, pode ser utilizada modelagem matemática do movimento gravitacional de suspensões particuladas em proveta ou modelagem matemática da sedimentação gravitacional em proveta, que não é simplesmente um problema puro de sedimentação. A modelagem é mais complexa, pois envolve sub-regiões que variam com o tempo e são delimitadas por interfaces em movimento, sendo que em cada região a porosidade e as velocidades satisfazem um sistema de equações diferenciais parciais.

Neste trabalho, em uma dimensão, duas soluções exatas, de uma forma de sistema do trabalho de Silva e Jesus [4], determinadas por Libório et al. [5], são inseridas em modelagem matemática do movimento gravitacional de suspensões particuladas em proveta, uma na região de sedimentação livre e outra na região de transição. Além disso, é usada a base preliminar de informações estabelecida por Libório et al. [5].

As soluções exatas e os movimentos da onda de aceleração e das interfaces, juntamente com dados experimentais da literatura de altura versus tempo da interface superior descendente do próprio teste de proveta, são usados para o cálculo de parâmetros envolvidos em equações propostas para as partes constitutivas da força de interação e de cada tensor tensão. Com isso, é determinada a diferença de pressões arbitrárias.

São mostrados resultados numéricos do cálculo dos parâmetros envolvidos nas equações do modelo, usando-se como ponto de partida a determinação dos parâmetros básicos velocidade da onda de aceleração, ponto de encontro da onda com a interface superior descendente e ponto de encontro das interfaces.

\section{ELEMENTOS BÁSICOS}

Para uma suspensão particulada, considerada como um meio poroso saturado com fluido e modelado pela teoria contínua de misturas sólido-fluido sem reações químicas, a densidade do constituinte fluido $\rho_{1}$ e a densidade do constituinte sólido $\rho_{2}$ são dadas por

$$
\rho_{1}=\varepsilon \rho_{f} \quad \text { e } \quad \rho_{2}=(1-\varepsilon) \rho_{s}
$$

nas quais, $\rho_{f}$ e $\rho_{s}$ são, respectivamente, as densidades do fluido e do sólido puros e $\varepsilon$ é a função porosidade [4].

Em uma dimensão, as equações do movimento de uma suspensão particulada, com restrição de incompressibilidade, oriundas dos balanços de massa e quantidade de movimento linear, são expressas pelas Equações (1), $\operatorname{com} \rho_{s}$ e $\rho_{f}$ constantes, e as equações relatadas a seguir [4].

$$
\begin{array}{llcc}
\frac{\partial \varepsilon}{\partial t}+\frac{\partial\left(\varepsilon V_{1}\right)}{\partial y}=0 & \text { e } \quad \frac{\partial \varepsilon}{\partial t}-\frac{\partial}{\partial y}\left[(1-\varepsilon)\left(V_{2}\right)\right]=0 & \\
\rho_{1} a_{1}=-\varepsilon & \frac{\partial P_{f}}{\partial y}+\frac{\partial \sigma_{1}}{\partial y}+m+\rho_{1} g_{1} & \text { com } & a_{1}=\frac{\partial V_{1}}{\partial t}+V_{1} \frac{\partial V_{1}}{\partial y} \\
\rho_{2} a_{2}=-(1-\varepsilon) \frac{\partial P_{s}}{\partial y}+\frac{\partial \sigma_{2}}{\partial y}-m+\rho_{2} g_{2} & \text { com } & a_{2}=\frac{\partial V_{2}}{\partial t}+V_{2} \frac{\partial V_{2}}{\partial y} \\
\frac{\partial \varepsilon}{\partial t} \frac{\partial P_{s f}}{\partial y}=\frac{\partial \varepsilon}{\partial y} \frac{\partial P_{s f}}{\partial t} & \mathrm{com} \quad P_{s f}=P_{s}-P_{f} &
\end{array}
$$

onde $t$ é a variável tempo e $y$ é a coordenada espacial. $V_{1}, V_{2}, a_{1}$ e $a_{2}$, são, respectivamente, as velocidades e as acelerações dos constituintes fluido e sólido. $P_{f}$ e $P_{S}$ são pressões arbitrárias. $\sigma_{1} \mathrm{e}$ $\sigma_{2}$ são, respectivamente, as partes constitutivas dos tensores tensão dos constituintes fluido e sólido. 
$m$ é a parte constitutiva da força de interação. As grandezas vetoriais $g_{1}$ e $g_{2}$ são as respectivas forças de campo. $P_{s f}$ é a diferença de pressões arbitrárias que satisfaz a Equação (5) determinada pela teoria de Silva e Jesus [4].

As Equações (3), (4) e (5) fornecem a equação de interseção

$$
\rho_{s} a_{2}-\rho_{f} a_{1}=-\frac{\partial P_{s f}}{\partial y}+\frac{1}{1-\varepsilon} \frac{\partial \sigma_{2}}{\partial y}-\frac{1}{\varepsilon} \frac{\partial \sigma_{1}}{\partial y}-\frac{m}{\varepsilon(1-\varepsilon)}+\rho_{s} g_{2}-\rho_{f} g_{1}
$$

\section{METODOLOGIA}

\subsection{SOLUÇÕES EXATAS}

Do trabalho de Libório et al. [5] são determinadas duas soluções exatas para o sistema formado pelas Equações (1)-(6). A primeira é a solução constante caracterizada por

$$
\frac{m}{\varepsilon(1-\varepsilon)}=\rho_{s} g_{2}-\rho_{f} g_{1} \quad \text { e } \quad \varepsilon, V_{1}, V_{2} \text { e } P_{s f} \text { constantes }
$$

A construção da segunda solução começa com a observação de que a consideração

$$
P_{s f}=P_{s f}(y, t)=P_{s f}(\varepsilon) \quad \operatorname{com} \quad \varepsilon=\varepsilon(y, t)
$$

satisfaz a Equação (5). Isso motiva a procura de outras soluções exatas sob as suposições

$$
V_{1}=V_{1}(y, t)=V_{1}(\varepsilon) \quad \text { e } \quad V_{2}=V_{2}(y, t)=V_{2}(\varepsilon)
$$

de tal modo que derivadas da porosidade e das velocidades também sejam funções da porosidade, no sentido da composição de funções expressa nas Equações (8) e (9). Dessa forma, suposições constitutivas generalizadas para as partes constitutivas da força de interação e de cada tensor tensão também podem ser colocadas na forma

$$
m=m(y, t)=m(\varepsilon) ; \quad \sigma_{1}=\sigma_{1}(y, t)=\sigma_{1}(\varepsilon) ; \quad \sigma_{2}=\sigma_{2}(y, t)=\sigma_{2}(\varepsilon)
$$

Com isso, usando como base as Equações (8)-(10), a segunda solução exata determinada por Libório et al. [5] pode ser colocada na forma

$$
\begin{array}{lcc}
V_{2}=w_{0}-\left(u_{o}+w_{0}\right) \frac{1-\varepsilon_{0}}{1-\varepsilon} & \text { e } & V_{1}=w_{0}+\frac{u_{0}-\varepsilon_{0}\left(u_{o}+w_{0}\right)+\varphi}{\varepsilon} \\
\int A(\varepsilon) d \varepsilon=y-w_{0} t+\text { constante } ; & A(\varepsilon) \frac{\partial \varepsilon}{\partial y}=1 \\
G(\varepsilon) A(\varepsilon)=B(\varepsilon)-\frac{d P_{s f}}{d \varepsilon}-Q(\varepsilon) ; & G(\varepsilon)=\frac{m}{\varepsilon(1-\varepsilon)}-\left(\rho_{s} g_{2}-\rho_{f} g_{1}\right) \\
B(\varepsilon)=\frac{1}{1-\varepsilon} \frac{d \sigma_{2}}{d \varepsilon}-\frac{1}{\varepsilon} \frac{d \sigma_{1}}{d \varepsilon} ; & Q(\varepsilon)=\rho_{s}\left[\left(\frac{w_{0}-V_{2}}{1-\varepsilon}\right)^{2}\right]+\rho_{f}\left[\left(\frac{w_{0}-V_{1}}{\varepsilon}\right)^{2}\right]
\end{array}
$$

onde $w_{0}$ e $u_{0}$ são velocidades constantes, $A(\varepsilon)$ é uma função arbitrária da porosidade com unidade de comprimento e $\varphi$ é uma constante arbitrária.

\subsection{MODELAGEM MATEMÁTICA}

Esta parte utiliza a estrutura desenvolvida por Libório et al. [5] para o movimento gravitacional de suspensões particuladas em proveta (sedimentação gravitacional em proveta), caracterizado de acordo com a Figura 1. 


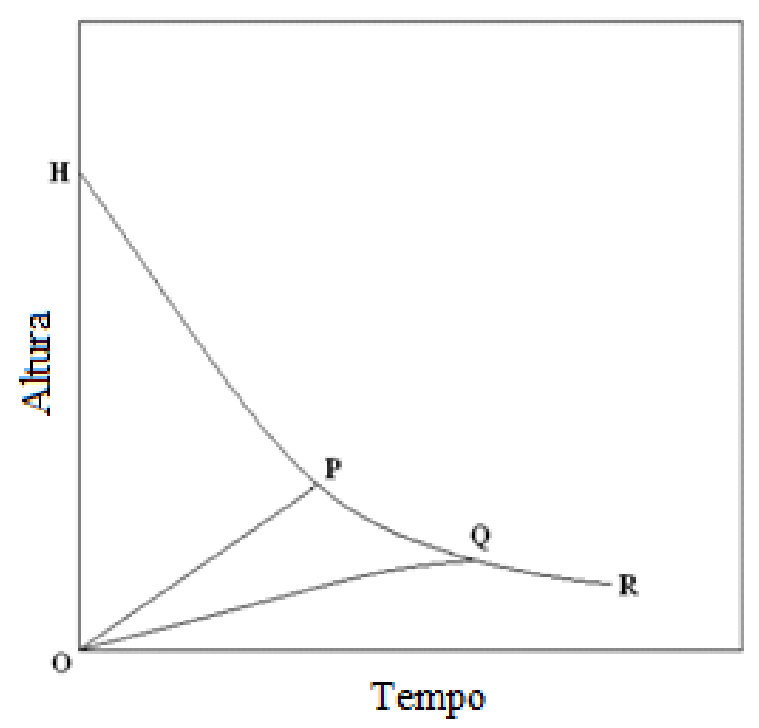

Figura 1: Gráfico representativo do processo de sedimentação em proveta.

No gráfico da Figura 1, HPQR até a altura $\mathrm{H}$ corresponde à região de líquido puro, $\mathrm{OPHO}$ corresponde à região de sedimentação livre, $\mathrm{OPQO}$ corresponde à região de transição e a região limitada por $\mathrm{OQR}$ e o eixo dos tempos corresponde à região de compressão. As curvas $\mathrm{HPQR}, \mathrm{OP}$ e OQ correspondem, respectivamente, às alturas da interface superior descendente, da onda de aceleração e da interface inferior ascendente. A introdução da região de transição OPQO foi motivada pelo trabalho de Kynch [6] e a revisão da sua teoria feita por Tiller [7] e estendida pelos trabalhos de Fitch [8] e Font [9]. A onda de aceleração, que não precisa ser visível, corresponde à propagação inicial da onda de concentração, a única que parte do fundo da proveta.

Em relação a cada curva do gráfico da Fig. 1, podem ser escritas as seguintes notações:

$$
x=x(t) \quad(0 \leq t<\infty) ; \quad h=h(t) \quad\left(0 \leq t \leq t_{0}\right) ; \quad z=z(t) \quad\left(0 \leq t \leq t_{c}\right)
$$

onde $x$ é a altura da interface superior descendente, $h$ é a altura da onda de aceleração e $z$ é a altura da interface inferior ascendente. $\mathrm{O}$ tempo $t_{0}$ indica o instante de encontro da interface superior descendente com a onda de aceleração, enquanto que o tempo $t_{c}$ indica o instante de encontro das duas interfaces. As alturas satisfazem as condições iniciais e as condições de encontro, respectivamente,

$$
x(0)=H ; \quad h(0)=z(0)=0 ; \quad x\left(t_{0}\right)=h\left(t_{0}\right) \equiv x_{0} ; \quad x\left(t_{c}\right)=z\left(t_{c}\right) \equiv x_{c}
$$

nas quais $H$ é a altura inicial da suspensão na proveta. Além disso

$$
\varepsilon(y, 0)=\varepsilon_{0}=\text { constante } \quad 0 \leq y \leq H ; \quad g_{1}=g_{2}=-g
$$

onde $\varepsilon_{0}$ é a porosidade inicial, $g$ é a aceleração da gravidade, a coordenada espacial $y$ está orientada para cima e $y=0$ representa o fundo da proveta. Desse modo, das Equações (2), (13) e (17), das condições de salto e das condições de velocidades nulas no fundo da proveta

$$
\varepsilon V_{1}+(1-\varepsilon) V_{2}=0 \quad 0 \leq y \leq x(t) ; \quad G(\varepsilon)=\frac{m}{\varepsilon(1-\varepsilon)}+\left(\rho_{s}-\rho_{f}\right) g
$$

\subsubsection{Uso das soluções exatas}

A primeira solução e a segunda solução são inseridas, respectivamente, nas regiões de sedimentação livre e de transição. Assim, das Equações (7), (11)-(18) e de Libório et al. [5]

$$
\varepsilon(y, t)=\varepsilon_{0} \quad 0 \leq t \leq t_{0} ; \quad V_{2}(y, t)=-u_{0} \quad 0<t \leq t_{0} ; \quad G\left(\varepsilon_{0}\right)=0
$$

para a região de sedimentação livre e 


$$
\begin{array}{lll}
V_{2}=w_{0}-\left(u_{0}+w_{0}\right) \frac{1-\varepsilon_{0}}{1-\varepsilon} & \text { e } \quad V_{1}=w_{0}+\frac{u_{0}-\varepsilon_{0}\left(u_{0}+w_{0}\right)}{\varepsilon} & 0<t \leq t_{c} \\
\int_{\varepsilon}^{\varepsilon_{0}} A(\xi) d \xi=w_{0} t-y & 0 \leq t \leq t_{c} \\
Q(\varepsilon)=\left(1-\varepsilon_{0}\right)^{2}\left[\rho_{s} \frac{\left(u_{0}+w_{0}\right)^{2}}{(1-\varepsilon)^{3}}+\frac{\rho_{f}}{\varepsilon^{3}}\left(\frac{w_{0}}{\left(1-\varepsilon_{0}\right)}-\left(u_{0}+w_{0}\right)\right)^{2}\right]
\end{array}
$$

para a região de transição, juntamente com as Equações (13) e (14).

De acordo com Libório et al. [5], da compatibilização das Equações (15), (16), (19)-(21) e das condições de salto através da onda de aceleração e da interface superior descendente

$$
\begin{array}{lll}
h=h(t)=w_{0} t ; & x=H-u_{0} t \quad 0 \leq t \leq t_{0} ; \quad x_{0}=w_{0} t_{0} \\
\left(u_{0}+w_{0}\right) t_{0}=H ; & \left(u_{0}+w_{0}\right) x_{0}=H w_{0} \\
\int_{\varepsilon^{-}}^{\varepsilon_{0}} A(\varepsilon) d \varepsilon=w_{0} t-x & \text { e } \quad \frac{d x}{d t}=w_{0}-\left(u_{0}+w_{0}\right) \frac{1-\varepsilon_{0}}{1-\varepsilon^{-}} \quad t_{0} \leq t \leq t_{c} \\
\left(w_{0} t-x\right)^{2}=2 N^{-}\left(x+u_{0} t-H\right) & t_{0} \leq t \leq t_{c} ; \quad N^{-}=N\left(\varepsilon^{-}\right)
\end{array}
$$

para as alturas da onda de aceleração e da interface superior descendente, respectivamente, em tais intervalos, onde $w_{0}$ e $u_{0}$ são, respectivamente, as velocidades da onda de aceleração e de sedimentação livre, $\varepsilon^{-}$é a porosidade no lado inferior de tal interface e $N(\varepsilon)$ é outra função com unidade de comprimento definida por

$$
2\left(\int_{\varepsilon}^{\varepsilon_{0}}\left(\varepsilon_{0}-\xi\right) A(\xi) d \xi\right) N(\varepsilon)=\left(1-\varepsilon_{0}\right)\left(\int_{\varepsilon}^{\varepsilon_{0}} A(\xi) d \xi\right)^{2}
$$

As Equações (23)-(26) mostram que tudo depende dos parâmetros básicos $u_{0}, w_{0}$ e do ponto $\left(x_{c}, t_{c}\right)$. Eles são determinados com o uso de pontos experimentais $(x, t)$ da interface superior descendente do próprio teste de proveta, como detalhado a seguir.

A velocidade de sedimentação livre $u_{0}$ é determinada da segunda relação das Equações (23). A velocidade da onda de aceleração $w_{0}$ é o mínimo positivo da função $w$

$$
w=\frac{u_{0} x^{2}}{2 H(H-x)-(2 H-x) u_{0} t} \quad \text { com } \quad 2 H(H-x)-(2 H-x) u_{0} t>0
$$

obtido pela substituição de pontos experimentais $(x, t)$ na relação (28).

$\mathrm{O}$ encontro das interfaces é o ponto $\left(x_{c}, t_{c}\right)$ onde ocorre o mínimo positivo da função $W$

$$
W=\frac{u_{0} x}{2(H-x)-u_{0} t} \quad \text { com } \quad 2(H-x)-u_{0} t>0
$$

obtido pela substituição de pontos experimentais $(x, t)$ na relação (29).

\subsubsection{Equações propostas para as grandezas constitutivas}

Para a parte constitutiva da força de interação é adotada a equação

$$
m=(1-\varepsilon) R\left(V_{2}-V_{1}\right) \quad \text { com } \quad R=R(\varepsilon)
$$

onde $R$ é a resistividade. Com isso, segue das as Equações (18) e (30) que

$$
m=\frac{(1-\varepsilon) R V_{2}}{\varepsilon}
$$

Então, das Equações (18), (19) e (31),

$$
u_{0}=\frac{\varepsilon_{0}{ }^{2}\left(\rho_{s}-\rho_{f}\right) g}{R_{0}} \quad \text { com } \quad R_{0}=R\left(\varepsilon_{0}\right)
$$

A Equação (32) motiva a introdução de uma velocidade $u$ dada por 


$$
u=u(\varepsilon)=\frac{\varepsilon^{2}\left(\rho_{s}-\rho_{f}\right) g}{R}
$$

Dessa forma, as Equações (32) e (33) fornecem a relação

$$
u=\frac{R_{0}}{R}\left(\frac{\varepsilon}{\varepsilon_{0}}\right)^{2}
$$

Baseado nas Equações (32)-(34) são adotadas as seguintes equações

$$
u=u_{o}\left(\frac{\varepsilon}{\varepsilon_{0}}\right)^{M} \quad \text { e } \quad R=R_{0}\left(\frac{\varepsilon_{0}}{\varepsilon}\right)^{M-2}
$$

onde $M$ é uma constante. Além disso, as Equações (20), (32) e (33) mostram que

$$
u\left(\varepsilon_{0}\right)=-V_{2}\left(\varepsilon_{0}\right)=u_{0}
$$

Isso sugere transferência de informações da velocidade $V_{2}$ para a velocidade $u$. Assim, para o cálculo de $M$, é adotada a condição

$$
\frac{d u}{d \varepsilon}\left(\varepsilon_{0}\right)=-\frac{d V_{2}}{d \varepsilon}\left(\varepsilon_{0}\right)
$$

Logo, das Equações (18), (20), (31)-(37) obtém-se que

$$
M=\frac{\varepsilon_{0}\left(u_{0}+w_{0}\right)}{u_{0}\left(1-\varepsilon_{0}\right)} \quad \text { e } \quad \frac{d G}{d \varepsilon}\left(\varepsilon_{0}\right)=0
$$

e as Equações (13), (19) e (38) determinam as condições

$$
B\left(\varepsilon_{0}\right)-\frac{d P_{s f}}{d \varepsilon}-Q\left(\varepsilon_{0}\right)=0 \quad \text { e } \quad \frac{d B}{d \varepsilon}\left(\varepsilon_{0}\right)-\frac{d^{2} P_{s f}}{d \varepsilon^{2}}-\frac{d Q}{d \varepsilon}\left(\varepsilon_{0}\right)=0
$$

Para as partes constitutivas de cada tensor tensão são adotadas as equações

$$
\sigma_{1}=2 \mu \frac{\partial V_{1}}{\partial y} \quad \text { e } \quad \sigma_{2}=\frac{\sigma_{0}}{2}\left(1+\left(\frac{1-\varepsilon_{0}}{1-\varepsilon}\right)^{\beta}\right) ; \quad \sigma_{0}=\sigma_{2}\left(\varepsilon_{0}\right)
$$

onde $\mu, \sigma_{0}$ e $\beta$ são constantes. Com isso, das Equações (12), (14), (20) e (40)

$$
\begin{array}{llrl}
B(\varepsilon)= & \frac{\beta\left(2 \sigma_{2}-\sigma_{0}\right)}{2(1-\varepsilon)^{2}}+\frac{2 \mu \tau}{\varepsilon^{4}}\left(\frac{\varepsilon}{A} \frac{d A}{d \varepsilon}+2\right) & \text { com } & \tau=\frac{\varepsilon_{0}\left(u_{0}+w_{0}\right)-u_{0}}{A} \\
\frac{d B}{d \varepsilon}(\varepsilon)=\frac{\beta(\beta+2)}{2(1-\varepsilon)^{3}}\left(2 \sigma_{2}-\sigma_{0}\right)+\frac{2 \mu \tau}{\varepsilon^{4}}\left[-\frac{8}{\varepsilon}-\frac{5}{A} \frac{d A}{d \varepsilon}+\frac{\varepsilon}{A} \frac{d^{2} A}{d \varepsilon^{2}}-\frac{2 \varepsilon}{A^{2}}\left(\frac{d A}{d \varepsilon}\right)^{2}\right]
\end{array}
$$

\subsubsection{Diferença de pressões arbitrárias}

As funções comprimento $A(\varepsilon)$ e a diferença de pressões arbitrárias estão relacionadas nas Equações (13). Por serem arbitrárias, fixada uma, a outra sustenta o sistema de equações. Por isso, usando a Equação (27), podem ser estudados os casos para os quais

$$
\begin{aligned}
& A=A(\varepsilon)=A_{0}=\text { constante } ; \quad N=N^{-}=\text {constante }=N_{0}=\left(1-\varepsilon_{0}\right) A_{0} \\
& \frac{d P_{s f}}{d \varepsilon}\left(\varepsilon_{0}\right)=\frac{2 \mu \tau}{\varepsilon_{0}^{4}}-\frac{Q\left(\varepsilon_{0}\right)}{2} ; \quad \frac{d P_{s f}^{2}}{d \varepsilon^{2}}\left(\varepsilon_{0}\right)=-\frac{1}{2} \frac{d Q}{d \varepsilon}\left(\varepsilon_{0}\right)-\frac{8 \mu \tau}{\varepsilon_{0}^{5}} \quad \text { com } \quad P_{S f}=P_{S f}(\varepsilon)
\end{aligned}
$$

Então, segue das Equações (19), (22), (24), (26), para $t=t_{c},(27),(38)$, (40)-(44) que

$$
\begin{array}{ll}
\frac{\beta \sigma_{0}}{\left(1-\varepsilon_{0}\right)^{2}}+\frac{4 \mu \tau}{\varepsilon_{0}^{4}}=Q\left(\varepsilon_{0}\right) & \text { com } \quad \tau=\frac{\varepsilon_{0}\left(u_{0}+w_{0}\right)-u_{0}}{A_{0}} \\
\frac{\beta(\beta+2) \sigma_{0}}{\left(1-\varepsilon_{0}\right)^{3}}-\frac{16 \mu \tau}{\varepsilon_{0}^{5}}=\frac{d Q}{d \varepsilon}\left(\varepsilon_{0}\right) &
\end{array}
$$




$$
\begin{aligned}
& \frac{d Q}{d \varepsilon}\left(\varepsilon_{0}\right)=3\left(1-\varepsilon_{0}\right)^{2}\left[\rho_{s} \frac{\left(u_{0}+w_{0}\right)^{2}}{\left(1-\varepsilon_{0}\right)^{4}}-\frac{\rho_{f}}{\varepsilon_{0}^{4}}\left(\frac{w_{0}}{\left(1-\varepsilon_{0}\right)}-\left(u_{0}+w_{0}\right)\right)^{2}\right] \\
& x(t)=N_{0}+w_{0} t-\sqrt{2 N_{0}\left(u_{0}+w_{0}\right) t-N_{0}\left(2 H-N_{0}\right)}, t_{0} \leq t \leq t_{c} \\
& N_{0}=\frac{\left(w_{0} t_{c}-x_{c}\right)^{2}}{2\left(x_{c}+u_{0} t_{c}-H\right)}
\end{aligned}
$$

Da estrutura estabelecida acima, as Equações (13), (18), (20), (22) e (31) fornecem as relações

$$
\begin{aligned}
& P_{s f}=I_{B}-I_{Q}-A_{0} I_{G}+\text { cte } \quad \operatorname{com} \quad I_{B}=\frac{\beta\left(2 \sigma_{2}-\sigma_{0}\right)}{2(\beta+1)(1-\varepsilon)}-\frac{4 \mu \tau}{3 \varepsilon^{3}} \\
& I_{Q}=\left(1-\varepsilon_{0}\right)^{2}\left(\frac{\rho_{s}}{2}\left(\frac{u_{0}+w_{0}}{1-\varepsilon}\right)^{2}-\frac{\rho_{f}}{2 \varepsilon^{2}}\left(\frac{w_{0}}{\left(1-\varepsilon_{0}\right)}-\left(u_{0}+w_{0}\right)\right)^{2}\right) \\
& I_{G}=\left(R_{0}\left(\frac{w_{0} \varepsilon_{0}^{M-2}}{1-M} \varepsilon^{1-M}-\varepsilon_{0}^{M-2}\left(u_{0}+w_{0}\right)\left(1-\varepsilon_{0}\right) \int \frac{1}{(1-\varepsilon) \varepsilon^{M}} d \varepsilon\right)+\left(\rho_{s}-\rho_{f}\right) g \varepsilon\right)
\end{aligned}
$$

para a diferença de pressões arbitrárias, $\operatorname{com} M \neq 1$. Para $M=1$

$$
\begin{aligned}
& P_{s f}=I_{B}-I_{Q}-A_{0} I_{G}+\text { cte } \quad \text { com } \quad I_{B}=\frac{\beta\left(2 \sigma_{2}-\sigma_{0}\right)}{2(\beta+1)(1-\varepsilon)}-\frac{4 \mu \tau}{3 \varepsilon^{3}} \\
& I_{Q}=\left(1-\varepsilon_{0}\right)^{2}\left(\frac{\rho_{s}}{2}\left(\frac{u_{0}+w_{0}}{1-\varepsilon}\right)^{2}-\frac{\rho_{f}}{2 \varepsilon^{2}}\left(\frac{w_{0}}{\left(1-\varepsilon_{0}\right)}-\left(u_{0}+w_{0}\right)\right)^{2}\right) \\
& I_{G}=\left(\frac{R_{0}}{\varepsilon_{0}}\left(w_{0} \ln \varepsilon-\left(u_{0}+w_{0}\right)\left(1-\varepsilon_{0}\right)\left(\ln \frac{\varepsilon}{1-\varepsilon}\right)\right)+\left(\rho_{s}-\rho_{f}\right) g \varepsilon\right)
\end{aligned}
$$

\begin{tabular}{|c|c|c|c|}
\hline$t(\min )$ & $x(\mathbf{c m})$ & $w\left(\mathrm{~cm} \mathrm{~min} \min ^{-1}\right)$ & $W\left(\mathrm{~cm} \mathrm{~min}^{-1}\right)$ \\
\hline $\mathbf{0 , 0 0}$ & 31,00 & 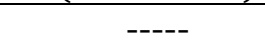 & ----- \\
\hline 1,25 & 18,19 & 13,86 & 14,14 \\
\hline 1,50 & 15,76 & 10,27 & 10,38 \\
\hline 1,75 & 13,52 & 8,14 & 7,90 \\
\hline 2,00 & 11,01 & 5,85 & 5,62 \\
\hline 2,25 & 8,75 & 4,56 & 4,06 \\
\hline 2,50 & 7,26 & 5,94 & 3,30 \\
\hline 2,75 & 7,12 & $-11,71$ & 3,60 \\
\hline
\end{tabular}

\section{RESULTADOS E DISCUSSÕES}

A Tabela 1 foi elaborada com base nos dados experimentais de Shannon et al. [10], com a velocidade de sedimentação livre $u_{0}$ obtida por meio da Equação (23). Ela mostra o cálculo da velocidade da onda de aceleração $w_{0}$ com base no mínimo positivo de $w$ dado pela Equação (28), como também o ponto de encontro das interfaces $\left(x_{c}, t_{c}\right)$ que é o ponto onde ocorre o mínimo positivo de $W$ dado pela Equação (29).

Tabela 1: Suspensão aquosa de esferas de vidro $\left(H=31 \mathrm{~cm}, \varepsilon_{0}=0,850, u_{0}=\right.$

Fonte: Elaboração própria com os dados de Shannon et al. [10]

A Tabela 1 e as Equações (24), (43) e (49) fornecem os resultados expressos na Tabela 2. 
Tabela 2: Suspensão aquosa de esferas de vidro $\left(H=31 \mathrm{~cm}, \varepsilon_{0}=0,850\right)$.

\begin{tabular}{cc}
\hline \multicolumn{2}{c}{ Sequência por linha do cálculo de $\boldsymbol{A}_{\mathbf{0}}$} \\
\hline$u_{0}=10,10 \mathrm{~cm} \mathrm{~min}^{-1}$ & $w_{0}=4,56 \mathrm{~cm} \mathrm{~min}^{-1}$ \\
$t_{0}=2,11 \mathrm{~min}^{2}$ & $x_{0}=9,64 \mathrm{~cm}$ \\
$t_{c}=2,50 \mathrm{~min}$ & $x_{c}=7,26 \mathrm{~cm}$ \\
$N_{0}=5,67 \mathrm{~cm}$ & $A_{0}=37,84 \mathrm{~cm}$ \\
\hline
\end{tabular}

Fonte: Elaboração própria com os dados de Shannon et al. [10]

Com os dados da Tabela 2 e as Equações (23) e (48) foi elaborada a Tabela 3, que mostra a comparação da altura teórica com a experimental.

Tabela 3: Suspensão aquosa de esferas de vidro $\left(H=31 \mathrm{~cm}, \varepsilon_{0}=0,850, u_{0}=\right.$

\begin{tabular}{ccc}
\multicolumn{3}{c}{$\left.10,10 \mathrm{~cm} \mathrm{~min}^{-1}\right)$} \\
$\boldsymbol{t}(\boldsymbol{m i n})$ & $\begin{array}{c}\boldsymbol{x}(\mathbf{c m}) \\
\text { experimental }\end{array}$ & $\begin{array}{c}\boldsymbol{x}(\boldsymbol{c m}) \\
\text { teórica }\end{array}$ \\
\hline $\mathbf{0 . 0 0}$ & 31,00 & 31,00 \\
$\mathbf{1 , 2 5}$ & 18,19 & 18,38 \\
$\mathbf{1 , 5 0}$ & 15,76 & 15,85 \\
$\mathbf{1 , 7 5}$ & 13,52 & 13,33 \\
$\mathbf{2 , 0 0}$ & 11,01 & 10,80 \\
$\mathbf{2 , 2 5}$ & 8,75 & 8,58 \\
$\mathbf{2 , 5 0}$ & 7,26 & 7,26 \\
\hline
\end{tabular}

Fonte: Elaboração própria com os dados de Shannon et al. [10]

Como da Tabela 3 os valores teóricos estão próximos dos experimentais, $A_{0}=37,84 \mathrm{~cm}$ é aceito. Com isso, a função comprimento fica determinada pela Equação (43) e é fundamental para obtenção de informações dos parâmetros $\sigma_{0}$ e $\beta$ com base nas Equações (45)-(49), cujos resultados estão expressos na Tabela 4.

Tabela 4: Suspensão aquosa de esferas de vidro $\left(H=31 \mathrm{~cm}, \varepsilon_{0}=0,850, \rho_{s}=2,45 \mathrm{~g} \mathrm{~cm}^{-3}\right.$,

$$
\begin{gathered}
\rho_{f}=\frac{\left.1 \mathrm{~g} \mathrm{~cm}^{-3}, \mu=0,6 \mathrm{~g} \mathrm{~cm}^{-1} \mathrm{~min}^{-1}\right)}{\text { Parâmetros }} \\
\hline M=8,23 \\
\beta=0,99 \\
R_{0}=365942,67 \mathrm{~g} \mathrm{~cm}^{-3} \mathrm{~min}^{-1} \\
\sigma_{0}=79,88 \mathrm{~g} \mathrm{~cm}^{-1} \mathrm{~min}^{-2} \\
\text { Fonte: Elaboração própria }
\end{gathered}
$$

Para efeito de validação, na Tabela 4 , os parâmetros $\sigma_{0}$ e $\beta$ foram determinados usando para $\mu \mathrm{o}$ valor da viscosidade da água.

Com os dados das Tabelas 2 e 4, a diferença de pressões arbitrárias fica determinada pelas Equações (50)-(55).

Se o valor de $A_{0}$ calculado não produz a altura teórica compatível com a altura experimental, a Equação (43) não é adequada para o estudo da suspensão particulada. Neste caso, deve ser proposta outra forma de equação no lugar da Equação (43) para a função comprimento, o que implica em mudanças nas Equações (43) e (48).

\section{CONCLUSÃO}

Equações estabelecidas neste trabalho, juntamente com dados experimentais, coletados da literatura, de altura versus tempo da interface superior descendente do próprio teste de proveta, mostram uma aplicação da base preliminar de informações estabelecida por Libório et al. (2015). 
De fato, a base fornece elementos para o estudo de grandezas constitutivas e arbitrárias de uma suspensão particulada com restrição de incompressibilidade. Pois, a partir dela podem ser determinados parâmetros envolvidos em equações propostas para as partes constitutivas da força de interação e de cada tensor tensão, como também para a função diferença de pressões arbitrárias.

\section{NOMENCLATURA}

$\varepsilon \quad$ Porosidade

$\rho_{1} \quad$ Densidade do constituinte fluido

$\rho_{2}$ Densidade do constituinte sólido

$\rho_{f} \quad$ Massa específica do fluido puro

$\rho_{s} \quad$ Massa específica do sólido puro

$V_{1}$ Velocidade do constituinte fluido

$V_{2}$ Velocidade do constituinte sólido

$t$ Variável tempo

$y$ Coordenada espacial

$P_{f} \quad$ Pressão do constituinte fluido

$P_{S} \quad$ Pressão do constituinte sólido

$P_{s f}$ Diferença de pressões arbitrárias

$\varphi$ Função do tempo

$a_{1}$ Aceleração do constituinte fluido

$a_{2}$ Aceleração do constituinte sólido

$\sigma_{1}$ Parte constitutiva do tensor tensão para o fluido

$\sigma_{2}$ Parte constitutiva do tensor tensão para o sólido

$m$ Parte constitutiva da força de interação

$g_{1}$ Força de campo sobre o fluido

$g_{2}$ Força de campo sobre o sólido

$H$ Altura inicial da suspensão na proveta

$x$ Altura da interface superior descendente

$h$ Altura da onda de aceleração

$z \quad$ Altura da interface inferior ascendente

$t_{0}$ Instante de encontro da interface superior descendente com a onda de aceleração

$t_{c}$ Instante de encontro das duas interfaces

$x_{0}$ Altura de encontro da interface superior descendente com a onda de aceleração

$x_{c} \quad$ Altura de encontro das duas interfaces

$\varepsilon_{0} \quad$ Porosidade inicial

$u_{0}$ Velocidade de sedimentação livre

$w_{0}$ Velocidade da onda de aceleração

$A$ Função comprimento

$B$ Função da porosidade

$Q$ Função da porosidade

$G$ Função da porosidade

$g$ Aceleração da gravidade

$N$ Comprimento função da porosidade

$w$ Função velocidade

W Função velocidade

$N$ Função comprimento

$R$ Resistividade

$M$ Constante admensional

$\mu$ Viscosidade da água

$\sigma_{0}$ Tensão inicial no sólido

$\beta$ Constante adimensional 


\section{REFERÊNCIAS BIBLIOGRÁFICAS}

1. Cremasco MA. Operações unitárias em sistemas particulados e fluidomecânicos - 2a edição. São Paulo. Blucher; 2014.

2. Hernando L, Omari A, Reungoat D. Experimental investigation of batch sedimentation of concentrated bidisperse suspensions. Powder Technology. 2015 May;(275) 273-279, doi: 10.1016/j.powtec.2015.01.069.

3. Tien C, Ramarao BV, Yasarla R. A blocking model of membrane filtration. Chemical Engineering Science. 2014 May;111: 421-431, doi: 10.1016/j.ces.2014.01.022.

4. Silva AS, Jesus E. On arbitrary quantities in a porous media with incompressibility constraint. International Review of Chemical Engineering. 2013 Oct;5(6): 429-434.

5. Libório DO, Santos EJ, Santana PL, Silva CF, Silva AS. Uma base preliminar de informações para o estudo do movimento de uma suspensão particulada com restrição de incompressibilidade. Scientia Plena. 2015 Out;11(10) 104201-1-14, doi: 10.14808/sci.plena.2015.104202.

6. Kynch GJ. A theory of sedimentation. Transactions of the Faraday Society. 1952 48: 166-176, doi: 10.1039/TF9524800166.

7. Tiller FM. Revision of Kynch sedimentation theory. AIChE Journal. 1981 Sep;27(5): 823-829, doi: 10.1002/aic.690270517.

8. Fitch B. Kynch theory and compression zones. AIChE Journal. 1983 Nov;29(6): 940-947, doi: 10.1002/aic.690290611.

9. Font R. Compression zone effect in batch sedimentation. AIChE Journal. 1988 Feb;34(2): 229-252, doi: 10.1002/aic.690340207.

10. Shannon PT, Dehaas RD, Stroupe EP, Tory EM. Batch and continuous thickening. Prediction of batch settling behavior from initial rate data with results for rigid spheres. Industrial \& Engineering Chemistry Fundamentals. 1964 Aug;3(3): 250-260, doi: 10.1021/i160011a014 